\section{It takes two}

Microtubule motors of the kinesin- 8 family depolymerize microtubules in a length-dependent manner, but the mechanism underlying this feedback of length on end dynamics has been unclear. Using single-molecule microscopy, Howard and colleagues studied the behavior of individual Kip3p (budding yeast kinesin-8) molecules on microtubules over a range of Kip3p concentrations. They found that single molecules walk towards the plus end, where they pause for $\sim 30 \mathrm{~s}$. Using quantum dot-labeled Kip3p molecules, the authors showed that Kip3p remains stationary while it pauses at the microtubule end and therefore does not continuously depolymerize microtubules. An increase in concentration of Kip3p leads to a decreased residence time of Kip3p molecules at the microtubule plus ends, suggesting that Kip3p molecules influence each other at microtubule ends. The end-residence time of Kip3p inversely correlates with the depolymerization rate, and the relation between them suggests a stoichiometry of one dissociating tubulin dimer for each dissociating Kip3p molecule. The observed stoichiometry and linear relation between flux of Kip3p to the plus end and depolymerization rate allowed them to formulate mathematically the so-called antenna model. According to this model, the number of motor molecules binding along a microtubule is proportional to its length, and high processivity ensures that all motors reach the microtubule end, where they cause depolymerization. The detachment of end-bound Kip3p accelerated by the end-reaching Kip3p molecules does not require depolymerization, suggesting a direct mechanical interaction between the end-bound and incoming Kip3p molecules. This 'bump off' mechanism leads to a coupling between the flux of Kip3p at the plus end and the depolymerization rate, which is crucial for length-dependent depolymerization. (Cell 138, 1174-1183, 2009)

\section{You must flip it}

In sharp contrast to the simplistic cartoons frequently used to depict cellular membranes in the scientific literature, the intracellular and extracellular faces of eukaryotic cells are highly complex environments. The two leaflets of the bilayer are composed of differing ratios of the various phospholipids, and this asymmetry is established by 'flippases', which actively transport specific phospholipids from the outer leaflet to the cytosolic face of the membrane. It was believed that one or more Type IV P-type ATPases (P4-ATPases) are responsible for this flippase activity in eukaryotic cells, but there has not been a clear demonstration that a purified P4-ATPase can act directly as a flippase in vitro. Zhou and Graham now report that purified Drs2p, a P4-ATPase from Saccharomyces cerevisiae, can actively transport a fluorescent analog of phosphatidylserine, an important component of the inner leaflet of eukaryotic membranes, in proteoliposomes. The putative flippase was highly selective, transporting the fluorescent analog of phosphatidylserine, but not fluorescent analogs of phosphatidylcholine or sphingomyelin, to the outer leaflet of the proteoliposome. Mass spectrometry analysis of the proteoliposomes indicated that a substoichiometric amount of the P4-ATPase $\beta$ subunit Cdc50p was also present; the authors determined that $\mathrm{Cdc50} \mathrm{p}$ was not directly responsible for the observed flippase activity, but it might still play some role in this process, perhaps by interacting with Drs2p. Additional experiments are needed to determine the exact role of Cdc50p in this process and the mechanism by which Drs2p is

Written by Angela K. Eggleston, Joshua M. Finkelstein \&

Arianne Heinrichs selective for phosphatidylserine. (Proc. Natl. Acad. Sci. USA published online, doi:10.1073/pnas.0904293106, 15 September 2009)

$J M F$

\section{Unstable at any age}

Eukaryotic ribosomal DNA (rDNA) loci exist as tandem repeats, but recombination can reduce the copy number, which would deleteriously affect translation. Yeast cells have evolved a counteracting mechanism to amplify these sequences, with the protein Fob1 playing a central role in maintaining rDNA copy number by stimulating recombination. Although these loci contain Fob1-binding sequences, which inhibit replication forks and act as 'hot spots' of amplification recombination, direct evidence that replication is required for amplification was lacking. Kobayashi and colleagues have now used a FOB1 mutant that only carries two copies of the rDNA locus to address this question. When one of the two replication origins (rARS(1)) was deleted and FOB1 was reintroduced, no amplification was observed, arguing that $\operatorname{rARS}(1)$ is required for blocking replication. When other origins were substituted for rARS(1), a more efficient origin caused increased copy amplification, whereas a less efficient origin showed lower amplification. Likewise, the level of extrachromosomal ribosomal DNA circles (ERCs) varied with the efficiency of replication initiation, allowing the authors to directly address the model that ERCs levels are negatively correlated with lifespan. In contrast to expectations from this model, both the high- and lowefficiency origin strains showed lifespan reduction, even though the latter strain has fewer ERCs than usual. rDNA instability was present in both 'altered origin' strains, and, in contrast to ERC number, rDNA instability correlated with lifespan. Finally, it was shown that unrelated episomes that are not rDNA-derived can also induce rDNA instability. These data suggest that the ERC model of aging must be revised, as it now seems that rDNA instability, and the presence of any extrachromosomal DNA, can shorten lifespan. (Mol. Cell 35, 683-693, 2009)

$A K E$

\section{A sense for destruction}

Cellular iron homeostasis in mammals is maintained by iron regulatory proteins (IRPS), which coordinate the posttranscriptional regulation of genes that function in iron homeostasis. Protein levels of IRP2 are in turn regulated in response to iron, but how IRP2 is degraded in iron-replete conditions is poorly understood. Two independent studies from teams led by Bruick and by Wohlschlegel now report the identification of an E3 ubiquitin ligase that targets IRP2 for degradation in an iron-dependent manner. The E3 ligase complex contains the F-box-containing substrate adaptor protein FBXL5, which is required for assembly of functional E3 ligase. Both teams show that the stability of FBXL5 is regulated by iron and oxygen levels, and they identify a putative iron-binding hemerythrin-like domain in its $\mathrm{N}$ terminus that might function as an iron and oxygen sensor. Mutational and spectroscopic analysis confirmed that the hemerythrin-like domain of FBXL5 binds iron and oxygen directly. FBXL5 binding to these ligands results in the formation of a functional E3 ligase complex that catalyzes the ubiquitylation and subsequent degradation of IRP2. Conversely, loss of iron and oxygen binding by the hemerythrin domain of FBXL5 renders it susceptible to degradation, hence precluding the assembly of the E3 ligase complex and allowing IRP2 to accumulate. These findings thus identify FBXL5 as a central iron sensor that regulates cellular iron homeostasis in mammals. (Science published online, doi:10.1126/science.1176326 and doi:10.1126/science.1176333, 17 September 2009) 